\title{
Cerebral Hemorrhagic Manifestation of Extra-Cardiac Infective Endocarditis: What Radiological Diagnostic Approach Should Be Taken?
}

\author{
Husam H Mansour ${ }^{1,2 *}$ (iD) \\ ${ }^{1}$ Radiology Department, Al-Shifa Hospital, Gaza, Palestine \\ ${ }^{2}$ Medical Imaging Department, Al-Azhar University, Gaza, Palestine
}

*Corresponding author: Husam H Mansour, Radiology Department, Al-Shifa Hospital, Gaza, Palestine; Medical Imaging Department, Al-Azhar University, Gaza, Palestine, Tel: 00970598913323

\begin{abstract}
Introduction: Infective endocarditis results from microbial infection of intracardiac structures and is highly fatal if untreated. The infection is typically clinically silent prior to the onset of cerebral hemorrhagic events.

Case description: A 56-year-old male with a history of mitral valve replacement presented to the emergency department complaining of vague symptoms combined with neurological and cardiac problems. The patient was admitted, and medical investigations were performed. Blood tests and cultures indicated that the patient suffered from infective endocarditis. Echocardiography showed the presence of vegetation on the mitral valve with features of infective endocarditis. Non-contrast brain CT revealed no abnormalities. The patient was administered antibiotic therapy. After two days, the patient's status deteriorated. A second non-contrast brain CT was performed and showed an intracerebral hemorrhage.

Discussion: Cerebral hemorrhages occur rarely in infective endocarditis. These hemorrhages are usually attributed to ruptured mycotic aneurysms. Early screening of neurological complications by cerebral CTA will improve the treatment decision and outcome in patients with infective endocarditis and decrease the mortality rate of these cases.
\end{abstract}

\section{Introduction}

Infective endocarditis is a disease with high rates of morbidity and mortality (9-30\%) [1]. A cardiac vessel wall becomes infected with bacteria. It is then digested, leading to the formation of a false aneurysm, which is highly prone to rupture [2].
Mycotic aneurysms, also known as infective aneurysms, are rare inflammatory neurovascular lesions that account for $0.7-6.5 \%$ of all intracranial aneurysms and carry a high mortality rate when ruptured [3]. Clinically manifest neurological complications that occur in infective endocarditis patients are mostly due to septic brain embolization and the formation of an intracranial mycotic aneurysm [4].

Here, I report a case of infective endocarditis in a patient who presented with neurological disorders representing acute stroke. I emphasize the advantages of cerebral CTA in the detection of pre-complications of infective endocarditis, which may decrease the mortality rate.

\section{Case Description}

The patient was a 56-year-old male with cardiac disease and histories of mitral valve replacement and bronchial asthma. The mitral valve replacement was a mechanical type placed in the past three years. The patient neglected anticoagulation drugs. He presented to the emergency department complaining of fever, chills, shortness of breath, a decreased appetite for many days, indistinct speech, headaches, visual changes, nausea, vomiting, and weakness in the extremities. The emergency physicians developed several differential diagnoses. Thus, it was necessary to admit the patient to the hospital and perform the necessary tests. Blood cultures showed microorganisms commonly found 


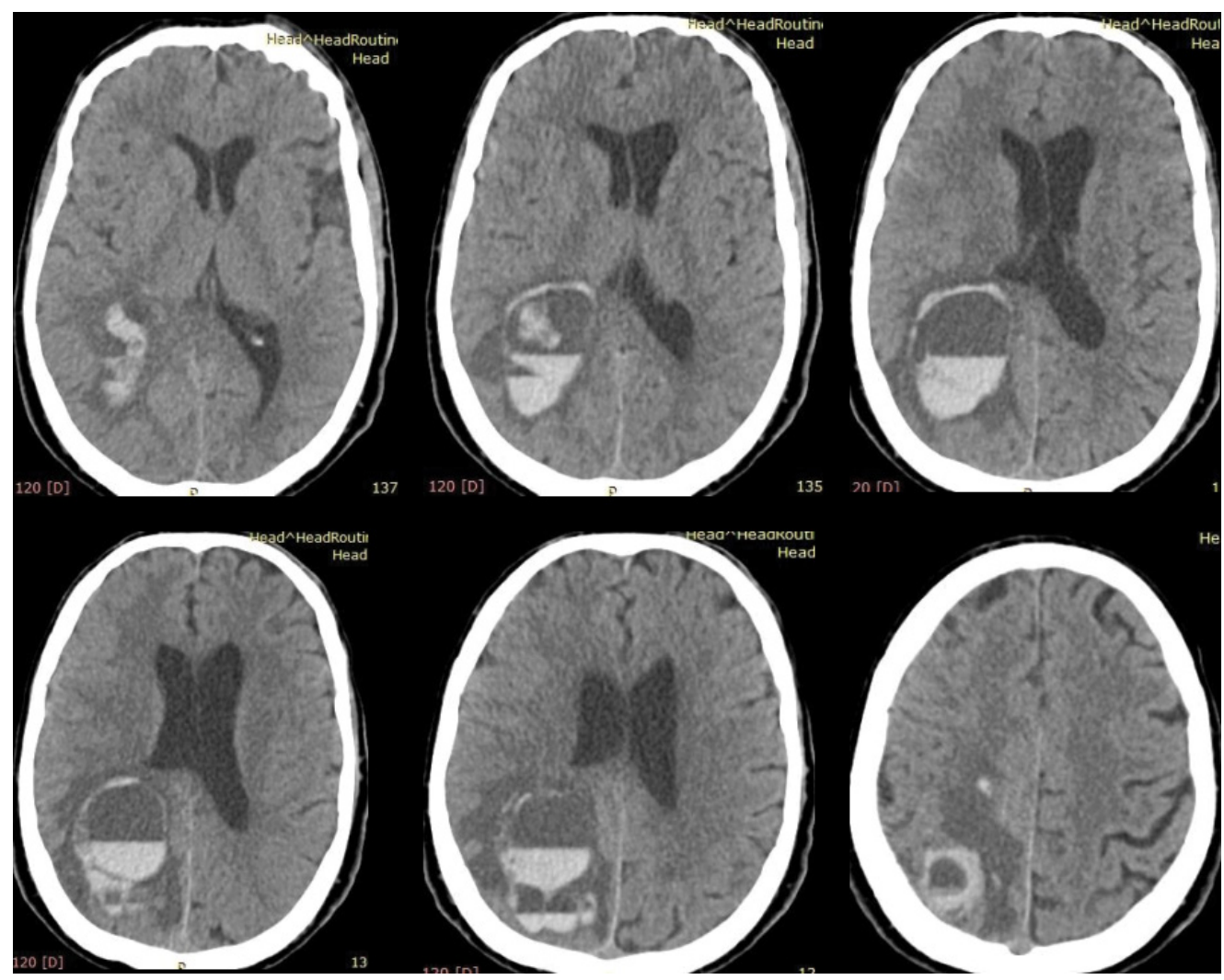

Figure 1: Non-contrast brain CT reveals RT parieto-occipital intracerebral hemorrhage associated with peripheral edema. Sedimentation level is seen, and that may be associated with recurrence of bleeding.

with endocarditis. Echocardiography revealed the presence of oscillating structures (vegetation) on the mitral valve with features of infective endocarditis. ECG was performed and was normal. Chest X-ray showed cardiomegaly without lung infiltrates, a thickened bronchial wall, and hilar enlargement. Therefore, the patient was treated conservatively with appropriate antibiotic therapy. Non-contrast brain CT identified no abnormalities. After two days, the patient's status worsened, with more neurological disorders such as total loss of consciousness, seizures, dizziness, loss of balance, confusion and disorientation. Another non-contrast brain CT was performed and showed an intracerebral hemorrhage (Figure 1).

\section{Discussion}

Neurological complications are the most common extra-cardiac manifestations in infective endocarditis and occur in approximately20-40\% of cases [5]. Mycotic aneurysms can develop in the cerebral or systemic circulation in these patients [6], and cerebral hemorrhage caused by stroke, a ruptured mycotic aneurysm, or septic necrotic arteritis with rupture of the vessel wall can cause neurologic complications.

Cerebral hemorrhage in the setting of infective endocarditis usually presents in the parenchyma or subarachnoid space. Parenchymal hemorrhage can be caused by rupture of an infectious aneurysm [5]. In a cohort of 1345 patients with infective endocarditis, hemorrhagic complications were strongly associated with Staphylococcus aureus infection and anticoagulant therapy. In such patients, a larger number of hemorrhagic events occurred, even after one week of antimicrobial therapy [7].

A study of 198 patients, which defined cerebral hemorrhage as primary intracerebral hemorrhage, hemorrhagic conversion of a prior ischemic infarct, or rupture of infectious aneurysm, found hemorrhage in $27 \%$ of patients [8].

Regarding the history of valve replacement, prosthetic valve endocarditis is associated with an increased rate of neurological complications. Mitral valve involvement and vegetation size have been considered important predictors of stroke in several studies [9-11].

Most symptomatic cerebral events occur within two weeks after the infective endocarditis diagnosis [12]. Furthermore, routine brain CT and CTA resulted in changes in the treatment plan in a significant proportion of patients with infective myocarditis, even those without the clinically evident neurological disease [13].

Unfortunately, in the current case only non-contrast brain CT was performed, as it is the first routine diagnostic scan for assessment of neurological disorders. Cerebral CTA was not performed.

There is no doubt that cerebral CTA is a noninvasive imaging technique used to evaluate cerebral vascular structures and plays an effective role in the early detection of infective endocarditis complications. Routine 
assessment of patients with infective endocarditis by cerebral CTA will change the treatment plans with a reduction in the mortality rate. This screening method is safe and may potentially improve the outcome of infective endocarditis patients. Importantly, we should be careful about Contrast-Induced Nephropathy (CIN) when performing cerebral CTA. A retrospective cohort study showed that the incidence of acute kidney injury (AKI) was $54.3 \%$ in infective endocarditis patients [14]. Implementation of a preventive strategy involving good hydration pre- and post-CT together with the use of low osmolality contrast media will reduce the risk of CIN [15]. Cardiologist consultation is highly recommended to avoid hypervolemia.

\section{Conclusion}

Neurological complications of infective endocarditis are serious complications and can cause significant morbidity and mortality. It is important to keep this disease entity in mind when encountering its complications because they often precede the diagnosis of infective endocarditis. Consequently, CTA of the brain and intracranial vessels should be performed in patients with neurological symptoms. The discovery of such complications demands careful consideration that may alter decision making regarding surgical management of the underlying disease. Finally, I recommend more studies to compare the implementation of routine screening by cerebral CTA versus the standard of care in infective endocarditis patients.

\section{Acknowledgements}

The author wishes to thank Prof. Thomas Foster of the University of Rochester, Rochester, NY USA for his careful reading of the manuscript, helpful suggestions, and English language editing.

\section{References}

1. Deprèle $C$, Berthelot $P$, Lemetayer $F$, Comtet $C$, Fresard $A$, et al. (2004) Risk factors for systemic emboli in infective endocarditis. Clin Microbiol Infect 10: 46-53.

2. McCready RA, Bryant MA, Divelbiss JL, Chess BA, Chitwood RW, et al. (2006) Arterial infections in the new millenium: An old problem revisited. Ann Vasc Surg 20: 590-595.

3. Kannoth S, Thomas SV (2009) Intracranial microbial aneurysm (infectious aneurysm): Current options for diagnosis and management. Neurocrit Care 11: 120-129.
4. Morris NA, Matiello M, Lyons JL, Samuels MA (2014) Neurologic complications in infective endocarditis: Identification, management, and impact on cardiac surgery. Neurohospitalist 4: 213-222.

5. Roder BL, Wandall DA, Espersen F, Frimodt-Moller N, Skinhoj P, et al. (1997) Neurologic manifestations in Staphylococcus aureus endocarditis: A review of 260 bacteremic cases in non-drug addicts. Am J Med 102: 379386.

6. Bisdas T, Teebken OE (2011) Mycotic or infected aneurysm? Time to change the term. Eur J Vasc Endovasc Surg 41: 570-571.

7. García-Cabrera E, Fernández-Hidalgo N, Almirante B, IvanovaGeorgieva R, Noureddine M, et al. (2013) Neurological complications of infective endocarditis risk factors, outcome, and impact of cardiac surgery: A multicenter observational study. Circulation 127: 22722284.

8. Sonneville R, Mirabel M, Hajage D, Comtet C, Fresard A, et al. (2011) Neurologic complications and outcomes of infective endocarditis in critically ill patients: The ENDOcarditeen reanimation prospective multicenter study. Crit Care Med 39: 1474-1481.

9. Cabell $\mathrm{CH}$, Pond KK, Peterson GE, Durack DT, Corey GR, et al. (2001) The risk of stroke and death in patients with aortic and mitral valve endocarditis. Am Heart J 142: 75-80.

10. Durante Mangoni E, Adinolfi LE, Tripodi MF, Andreana A, Gambardella M, et al. (2003) Risk factors for "major" embolic events in hospitalized patients with infective endocarditis. Am Heart J146: 311-316.

11. Dickerman SA, Abrutyn E, Barsic B, Bouza E, Cecchi $E$, et al. (2007) The relationship between the initiation of antimicrobial therapy and the incidence of stroke in infective endocarditis: An analysis from the ICE Prospective Cohort Study (ICE-PCS). Am Heart J 154: 1086-1094.

12. Vilacosta I, Graupner C, San Román JA, Sarriá C, Ronderos $R$, et al. (2002) Risk of embolization after institution of antibiotic therapy for infective endocarditis. J Am Coll Cardiol 39: 1489-1495.

13. Meshaal MS, Kassem HH, Samir A, Zakaria A, Baghdady $Y$, et al. (2015) Impact of routine cerebral CT angiography on treatment decisions in infective endocarditis. PLoS One 10: e0118616.

14. Von Tokarski F, Lemaignen A, Portais A, Fauchier L, Hennekinne F, et al. (2020) Risk factors and outcomes of early acute kidney injury in infective endocarditis: A retrospective cohort study. Int $\mathrm{J}$ Infect Dis 99: 421-427.

15. Mansour HH, Alajerami YS, Abushab MK (2021)Prophylaxis of Contrast-Induced Nephropathy in Intravascular Contrast Computed Tomography Procedures by Use of Low Osmolality Contrast Media and Good Hydration. Electron J Gen Med 18: em281. 